\title{
Rho kinase II interference by small hairpin RNA ameliorates 1-methyl-4-phenyl-1,2,3,6-tetrahydropyridine-induced parkinsonism in mice
}

\author{
QIONG ZHANG $^{1}$, YONG-FEI ZHAO ${ }^{1}$, JIAN-YING XI $^{1}$, WEN-BO YU $^{1}$ and BAO-GUO XIAO ${ }^{1-3}$ \\ ${ }^{1}$ Department of Neuroimmunity, Institute of Neurology, Huashan Hospital, Fudan University, Shanghai 200040; \\ ${ }^{2}$ Institute of Brain Science and State Key Laboratory of Medical Neurobiology, Fudan University, \\ Shanghai 200032; ${ }^{3}$ Institute of Neurology, Huashan Hospital, Shanghai 200040, P.R. China
}

Received August 26, 2015; Accepted May 12, 2016

DOI: $10.3892 / \mathrm{mmr} .2016 .5889$

\begin{abstract}
Novel therapeutic targets are required for the treatment of Parkinson's disease (PD). Previous studies suggest that the Rho/Rho-associated, coiled-coil-containing protein kinases (ROCKs) signaling pathway may be a promising therapeutic target in PD. To elucidate the importance of ROCKII in the pathogenesis of dopaminergic (DA) neuron loss and to investigate the efficacy of ROCK inhibitors in PD, ROCKII expression in the substantia nigra (SN) of mice was silenced through the injection of a lentivirus-based small hairpin RNA system. Empty lentivirus vectors served as controls. Mice were subsequently challenged with 1-methyl-4-phenyl-1,2,3,6-tetrahydropyridine (MPTP). The expression levels and activity of ROCKII were elevated in tyrosine hydroxylase-positive neurons and in cluster of differentiation (CD) 11b-positive microglia within the SN of MPTP-treated mice, which was accompanied by an increased level of expression of inducible nitric oxide synthase (iNOS) and activation of the Toll-like receptor (TLR)2/nuclear factor (NF)- $\mathrm{\kappa B}$ signaling pathway in M1 microglia. ROCKII interference (RI) significantly improved movement disorder and attenuated DA neuron loss induced by MPTP. In addition, RI inhibited the activation of M1 microglia in the SN, exhibiting reduced activity of the TLR2/NF- $\mathrm{KB}$ signaling pathway and decreased expression levels of iNOS and inflammatory factors, including interleukin (IL)-1 $\beta$ and IL- 6 . The results of the present study verify that ROCKII participates in the loss of DA neurons induced by MPTP and suggest that ROCKII inhibition may be a promising therapeutic target for PD.
\end{abstract}

Correspondence to: Professor Bao-Guo Xiao, Institute of Neurology, Huashan Hospital, 12 Middle Wulumuqi Road, Shanghai 200040, P.R. China

E-mail:bgxiao@shmu.edu.cn

Key words: Rho kinases, RNA interference, Parkinson's disease, microglia, neuro-inflammation

\section{Introduction}

Parkinson's disease (PD) is a common neuro-degenerative disorder that affects more than $0.1 \%$ of the population $>40$ years of age (1). The majority of PD patients present with slowed movement, rest tremors, rigidity and an abnormal posture. PD is associated with the progressive loss of dopaminergic (DA) neurons in the substantia nigra (SN), however, its etiology remains unclear. Neuro-inflammation has been implicated in the pathogenesis of PD (1). Currently available therapeutic agents provide symptomatic improvement; however, no disease-modifying treatments have been developed. Therefore, novel therapeutic targets for PD are required, and a promising target is the Rho/Rho-associated, coiled-coil-containing protein kinases (ROCKs) signaling pathway.

ROCKs are serine/threonine (Ser/Thr) protein kinases of which there are two isoforms, ROCKI and ROCKII, that are encoded by two separate genes. These two proteins share $92 \%$ homology in their kinase domains. ROCKI is primarily expressed in non-neuronal tissues, while ROCKII is predominantly expressed in the brain. Previous studies suggest that each isoform performs distinct functions. For instance, ROCKI expression is upregulated upon macrophage adhesion, whereas phagocytic activity is downregulated in ROCKII-depleted macrophages, but not in ROCKI-depleted cells $(2,3)$. In addition, ROCKs are markedly homologous with additional cyclic adenosine monophosphate (AMP)-dependent, cyclic guanosine monophosphate-dependent and protein kinase $\mathrm{C}$ kinases. ROCKs are involved in a wide variety of physiological and pathological processes, including differentiation, neurite growth and plasticity of neurons (4). In addition, ROCKs influence inflammatory responses by regulating migration and adhesion of leukocytes, phagocytosis of macrophages and secretion of pro-inflammatory cytokines (5-8).

ROCKs have been investigated in neuro-degenerative disorders, such as Alzheimer's disease (AD) (9). Previous studies have reported that inhibiting ROCK alleviated DA neuron loss induced by 1-methyl-4-phenyl-1,2,3,6-tetrahydropyridine (MPTP) in mice $(10,11)$. However, due to the substantial homology between ROCKs and additional kinases, currently used ROCKs inhibitors do not exclusively inhibit ROCKs. For 
example, fasudil has low selectivity for various other kinases, including protein kinase $\mathrm{N}$ (PKN), stress-induced kinase 1 (MSK1), mitogen-activated protein kinase 1b (MAPK1b) and protein kinase A (PKA) and moderate selectivity for AMP-activated protein kinase (AMPK) and phosphorylase kinase (PHK) (12). In addition, every ROCK inhibitor indiscriminately targets ROCKI and ROCKII. Consequently, it is considered to be impossible to accurately evaluate the role of ROCKs in PD using ROCKs inhibitors. The aim of the present study was to investigate the role of ROCKII in PD by constructing a lentivirus-based small hairpin (sh)RNA system that specifically interferes with the expression of ROCKII, in order to examine its neuro-protective effects during MPTP-induced neuron loss in the SN of mice.

\section{Materials and methods}

Animals. A total of 10 female C57BL/6 mice (age, 10-12 weeks; weight 22-25 g) were purchased from Shanghai Laboratory Animal Center (Shanghai, China). Mice were maintained at $25 \pm 2^{\circ} \mathrm{C}$ under $12 \mathrm{~h}$ light/dark cycles, and had access to food and water ad libitum. Animal procedures were performed in accordance with the International Council for Laboratory Animal Science guidelines, and the study received ethical approval from the Ethics Committee of Fudan University (Shanghai, China).

Antibodies. The mouse anti-mouse ROCKII monoclonal antibody (dilution, 1:1,000; cat. no. 610624; BD Biosciences, Franklin Lakes, NJ, USA), mouse anti-mouse inducible nitric oxide synthase (iNOS) monoclonal antibody (dilution, 1:200; cat. no. 610329; BD Biosciences), rabbit anti-mouse tyrosine hydroxylase (TH) polyclonal antibody (dilution, 1:1,000; cat. no. AB152; EMD Millipore, Billerica, MA, USA), mouse anti-mouse TH monoclonal antibody (dilution, 1:1,000; cat. no. MAB318; EMD Millipore), mouse anti-mouse cluster of differentiation (CD) 11b monoclonal antibody (dilution, 1:200; cat. no. 14-0112-82; eBioscience, Inc., San Diego, CA, USA), rabbit anti-mouse Toll-like receptor (TLR) 2 monoclonal antibody (dilution, 1:500; cat. no. 3268-1; Epitomics, Burlingame, CA, USA), rabbit anti-mouse phosphorylated TANK-binding kinase (p-TBK) 1 (Ser172) monoclonal antibody (dilution, 1:500; cat. no. 3300-1; Epitomics), rabbit anti-mouse $\mathrm{p}$-inhibitor of $\kappa \mathrm{B} \alpha(\mathrm{I} \kappa \mathrm{B} \alpha)$ polyclonal antibody (dilution, 1:500; cat. no. EPR6235 (2); Epitomics), rabbit anti-mouse GAPDH monoclonal antibody (dilution, 1:10,000; cat. no. 2251-1; Epitomics), rabbit anti-mouse p-IкB kinase (IKK) $\alpha / \beta$ (Ser176/180) monoclonal antibody (dilution, 1:500; cat. no. 2697; Cell Signaling Technology, Inc., Danvers, MA, USA), rabbit anti-mouse p-myosin phosphate target subunit (MYPT) 1 (Thr696) polyclonal antibody (dilution, 1:200; cat. no. 5163; Cell Signaling Technology, Inc.), rabbit anti-mouse p-myosin light chain (MLC) 2 (Ser19) polyclonal antibody (dilution, 1:200; cat. no. 3671; Cell Signaling Technology, Inc.) and rabbit anti-mouse p-nuclear factor (NF)- $\mathrm{B}$ p65 (Ser536) monoclonal antibody (dilution, 1:1,000; cat. no. 3033; Cell Signaling Technology, Inc.) were used for the purposes of the current study. The secondary antibodies used in this study were all purchased from Thermo Fisher Scientific, Inc., (Waltham, MA, USA), and included the following: Alexa Fluor 555-conjugated goat anti-mouse IgG (H+L; cat. no. A-21422); Alexa Fluor 555-conjugated goat anti-rabbit IgG $(\mathrm{H}+\mathrm{L}$; cat. no. A-21428); Alexa Fluor 488-conjugated goat anti-rabbit IgG (H+L; cat. no. A-11008); Alexa Fluor 488-conjugated goat anti-mouse IgG (H+L; cat. no A-11001); horseradish peroxidase (HRP)-conjugated goat anti-rabbit $\operatorname{IgG}(\mathrm{H}+\mathrm{L}$; cat. no. 31460); and HRP-conjugated goat anti-mouse IgG (H+L; cat. no. 31430 ).

BV2 microglia culture and 1-methyl-4-phenylpyridinium $(\mathrm{MPP}+)$ treatment. Mouse BV2 microglia cells were purchased from Shanghai Fuxiang Biotechnology Co., Ltd. (Shanghai, China) and cultured in Dulbecco's modified Eagle's medium (Gibco; Thermo Fisher Scientific, Inc.) supplemented with $10 \%$ fetal bovine serum (Gibco; Thermo Fisher Scientific, Inc.), $100 \mathrm{U} / \mathrm{ml}$ penicillin, and $100 \mu \mathrm{g} / \mathrm{ml}$ streptomycin at $37^{\circ} \mathrm{C}$ in a $95 \%$ humidified $5 \% \mathrm{CO}_{2}$ cell culture incubator. Cells were challenged with $0.5 \mathrm{mM}$ MPP+ (Sigma-Aldrich; Merck Millipore, Darmstadt, Germany) once for $24 \mathrm{~h}$ between 5 and 8 days following lentivirus transfection. BV2 cells were then collected and lysed in radioimmunoprecpitation (RIPA) lysis buffer (Beyotime Institute of Biotechnology, Shanghai, China) to extract total protein, and the lysates were centrifuged at $4{ }^{\circ} \mathrm{C}$ for $5 \mathrm{~min}$ at $10,000 \mathrm{x}$. The supernatant was stored at $-20^{\circ} \mathrm{C}$ until required.

Lentivirus-based shRNA system construction and screening. A lentivirus-based shRNA system was constructed by NeuronBiotech Co., Ltd. (Shanghai, China). Briefly, DH5 $\alpha$ competent cells (Takara Bio, Inc., Otsu, Japan) were used to produce the lentivirus vector, pLKD.CMV.GFP.U6, with U6 promoter as the transcriptional start site for shRNA. A 21-bp sequence containing a stem loop sequence was inserted into the vector to produce a valid shRNA that would interfere with ROCKII at the mRNA level (NM_009072.2). Five candidate lentiviruses targeting five unique sequences were constructed and an empty lentivirus vector served as a control. Lentiviruses were transfected into the mouse BV2 microglia cell line for screening. When cell density reached $\sim 30 \%$, lentiviruses were added into the culture medium with a multiplicity of infection of 20 for $24 \mathrm{~h}$. Protein levels and enzyme activity of ROCKII in the culture medium were measured to evaluate the shRNA interference systems once at 5-8 days following transfection.

Stereotaxic microinjection and RI in SN. Of the shRNA systems evaluated, two were confirmed to efficiently interfere with ROCKII expression. The two shRNA systems were combined at a ratio of 1:1 and injected into the $\mathrm{SN}$ of mice in the RI group, as previously described (13). Briefly, mice were anesthetized via intraperitoneal injection of $400 \mathrm{mg} / \mathrm{kg}$ chloral hydrate $(100 \mathrm{mg} / \mathrm{ml})$ and placed into the stereotaxic apparatus. Unilateral injection was performed into the right side of the brain $(3.0 \mathrm{~mm}$ posterior to bregma, $1.0 \mathrm{~mm}$ lateral to midline and $4.0 \mathrm{~mm}$ ventral to the dural surface). A total of $2 \mu \mathrm{l}$ lentivirus was injected with a microsyringe driven by a microdialysis pump at a rate of $0.2 \mu \mathrm{l} / \mathrm{min}$. Following microinjection, the syringe needle was left in place for $5 \mathrm{~min}$ prior to its withdrawal to reduce efflux of injected liquid along the injection tract. Control mice were injected with an empty vector system according to the same procedure. 
MPTP-induced parkinsonism. One week following microinjection, mice were challenged with intraperitoneal injections of MPTP (Sigma-Aldrich; Merck Millipore) dissolved in saline, which was administered every day for 7 successive days. The MPTP dose increased from $15 \mathrm{mg} / \mathrm{kg}$ on the first day and $20 \mathrm{mg} / \mathrm{kg}$ on the second day to a maximum of $30 \mathrm{mg} / \mathrm{kg}$ on the subsequent 5 days. Mice were maintained at $25-28^{\circ} \mathrm{C}$ following each injection.

Behavioral tests. The pole test (PT) and adhesive removal test (ART) were performed with minor modifications $(14,15)$. In the PT, mice were placed head-downward on the top of a $12-\mathrm{mm}$ thick, $80-\mathrm{cm}$ tall, vertical pole with a rough surface. The time for descent from the top surface to the floor was recorded. If the duration was $>120 \mathrm{sec}$, it was recorded as the default value, $120 \mathrm{sec}$. The investigator was blind to the animal grouping. In the ART, adhesive dots were placed on the plantar surface of the two forelimbs with equal pressure; the time for spot removal on each side was recorded. The default duration was set at $120 \mathrm{sec}$. Mice were trained daily for 2 continuous days ahead of the formal assessments. Tests were conducted daily and repeated for 3 continuous days prior to sacrifice.

Tissue processing. For protein extraction, mice were anesthetized via intraperitoneal injection of $400 \mathrm{mg} / \mathrm{kg}$ chloral hydrate $(100 \mathrm{mg} / \mathrm{ml}$, Sinopharm Chemical Reagent Co., Ltd., Shanghai, China) and perfused by intracardiac injection of $20 \mathrm{ml} \mathrm{0.9 \%}$ sodium chloride solution. The brain was carefully removed and homogenized in RIPA lysis buffer (Beyotime Institute of Biotechnology) supplemented with $1 \mathrm{mM}$ protease inhibitor (phenylmethanesulfonyl fluoride; Beyotime Institute of Biotechnology). The extracts were centrifuged at 12,000 x $g$ for $20 \mathrm{~min}$ at $4^{\circ} \mathrm{C}$. The supernatant was recovered and frozen at $-80^{\circ} \mathrm{C}$.

For histological analysis, mice were transcardially perfused with saline, followed by $4 \%$ paraformaldehyde (PFA) in phosphate-buffered saline (PBS). Brains were removed and dehydrated sequentially in 10, 20 and $30 \%$ (w/v) sucrose solution overnight at $4^{\circ} \mathrm{C}$. Brains were then embedded in Tissue-Tek O.C.T Compound (Sakura Finetek USA, Inc., Torrance, CA, USA), frozen in liquid nitrogen, sectioned at $10 \mu \mathrm{m}$ and stored at $-20^{\circ} \mathrm{C}$.

Immunofluorescence staining and DA neuron quantification. Sections were fixed with 4\% PFA at room temperature (RT) and washed thoroughly with PBS. Sections were then incubated for $30 \mathrm{~min}$ at RT in 5\% fetal bovine serum (Gibco; Thermo Fisher Scientific, Inc.) and 0.1\% Triton X-100 in PBS, and then incubated overnight at $4^{\circ} \mathrm{C}$ with the aforementioned primary antibodies diluted in PBS containing $1 \%$ fetal bovine serum and/or $0.1 \%$ Triton X-100. Following thorough washing with PBS, sections were incubated with the corresponding aforementioned secondary antibodies labeled with various fluorochromes at RT for $2 \mathrm{~h}$. Finally, sections were washed thoroughly and observed under a fluorescence microscope. Images were captured with DP controller software (version, 2.0; Olympus Corporation, Tokyo, Japan) and processed with DP manager (Olympus Corporation). Numbers of DA neurons were estimated by counting TH-positive cells from every twelfth $10-\mu \mathrm{m}$ section along the SN pars compacta (SNpc) with the aid of Image-Pro Plus software (version, 6.0; Media Cybernetics, Inc., Rockville, MD, USA) as previously described (16). Briefly, boundaries of the SNpc, the area of interest, were defined according to previously defined anatomical analysis of the mouse (17). Positive cells beyond the SNpc were excluded. Each RBG image was processed with a constant 'color-cube' segmentation setting and the same threshold, resulting in accurately defined foreground immunofluorescent staining. The total number of TH-positive cells in five sections of the same mouse brain was recorded and compared between shRNA and empty vector groups. The RBG image of each striatum section was processed with a constant 'color-cube' segmentation setting and at the same threshold and level. TH-positive areas were analyzed and the mean number of five sections for each brain was compared between shRNA and empty vector groups.

Western blotting and protein quantification. The protein concentration of brain sample extracts was determined using a bicinchoninic acid kit (Beyotime Institute of Biotechnology) and the whole protein extracts were diluted in RIPA lysis buffer (Beyotime Institute of Biotechnology) to a final protein concentration of $5 \mu \mathrm{g} / \mu \mathrm{l}$ prior to western blotting. Sample proteins $(10 \mu 1)$ were then separated by sodium dodecyl sulfate-polyacrylamide gel electrophoresis (8\%; Bio-Rad Laboratories, Inc., Hercules, CA, USA) at $80 \mathrm{~V}$ for $30 \mathrm{~min}$, before they were transferred to nitrocellulose membranes (EMD Millipore). After blocking with 3\% bovine serum albumin (w/v) for $1 \mathrm{~h}$ at RT, the membranes were incubated with primary antibodies overnight at $4^{\circ} \mathrm{C}$, followed by the appropriate HRP-conjugated secondary antibody for $2 \mathrm{~h}$ at RT. Protein bands were developed with chemiluminescent HRP substrate (EMD Millipore) and imaged with ChemiDOC XRS+ system (Bio-Rad Laboratories, Inc.) combined with Image Lab version 3.0 software (Bio-Rad Laboratories, Inc.). The signal intensity was quantified using Image Lab version 3.0 software. The density of each band was normalized to the band density of the constitutively expressed gene, GAPDH.

Enzyme-linked immunosorbent assays (ELISAs). The levels of interleukin (IL)-1 $\beta$, IL-6, and tumor necrosis factor (TNF)- $\alpha$ in the mouse brain were measured with the corresponding ELISA kits (cat. nos. 900-M47, 900-K50 and 900-M54, respectively; PeproTech, Inc., Rocky Hill, NJ, USA) according to the manufacturer's instructions, as described previously (18).

Statistical analysis. Data were analyzed with Student's $t$ test or Mann-Whitney $\mathrm{U}$ test and $\mathrm{P}<0.05$ was considered to indicate a statistically significant difference. All statistical analyses were performed using SPSS software version 12.0 (SPSS Inc., Chicago, IL, USA) and data are presented as the mean \pm standard error of the mean.

\section{Results}

Expression level and activity of ROCKII increased in the SNpc area of mice with MPTP-induced parkinsonism. The level of ROCKII expression in the brains of the MPTP-induced parkinsonism mice was $\sim 1.2$ times the level in the brains of the control mice $(\mathrm{P}<0.01$; Fig. 1A). The level of MYPT1 [a substrate 
Table I. Targeting sequences of five anti-ROCKII shRNAs.

\begin{tabular}{lc}
\hline shRNA & \multicolumn{1}{c}{ Target sequence } \\
\hline shR336 & GCTGGAAATTACCCTTACCAA \\
shR337 & CCCTGCAAAGTTTATTATGAT \\
shR338 & GCATCTCTTGAAGAAACAAAT \\
shR339 & GCTTGCACTGGATGCAATACA \\
shR340 & GCAGAGCAGTATTTCTCAACC
\end{tabular}

ROCKII, Rho/Rho-associated, coiled-coil-containing protein kinase II; shRNA, small hairpin RNA.

of ROCKII (19)] phosphorylated at Thr696 (p-MYPT1) in the brains of the MPTP-induced parkinsonism mice was $\sim 1.5$ times the level in the brains of the control mice $(\mathrm{P}<0.05$; Fig. 1A). The majority of p-MYPT1-positive cells exhibited a glia-like shape (Fig. 1B and C). These results demonstrated that ROCKII activation was involved in the MPTP-induced parkinsonism.

RI decreases the expression level and activity of ROCKII in vitro and in vivo. The schematic of lentivirus construction is presented in Fig. 2A and target sequences of five shRNAs are listed in Table I. ROCKII expression was inhibited to the greatest extent in shR340- and shR337-transfected cells, with decreases of $\sim 93 \%(\mathrm{P}<0.001)$ and $\sim 51 \%(\mathrm{P}<0.01)$, respectively, when compared with empty vector-transfected cells (Fig. 2B). The level of MLC phosphorylated at Ser19 (p-MLC), another substrate of ROCKII (12), was examined. shR337 and shR340 significantly decreased the level of p-MLC by $\sim 80 \%$ when compared with the empty vector ( $\mathrm{P}<0.01$; Fig. $2 \mathrm{~B})$. In addition, shR337 and shR340 markedly inhibited the expression and activity of ROCKII in the SN of MPTP-induced parkinsonism mouse brains following injection of combined lentiviruses into the SN region (Fig. 2C). These data demonstrate that shR337 and shR340 lentiviruses effectively inhibited the expression and activity of ROCKII in vitro and in the MPTP-induced parkinsonism.

RI attenuated movement disorder and DA neuron loss induced by MPTP. RI mice outperformed the control mice in the PT and ART tests. In the PT (Fig. 3A), RI mice moved from the top surface to the ground more rapidly than the control mice ( $6.9 \pm 0.6$ vs. $9.8 \pm 1.1 \mathrm{sec} ; \mathrm{P}<0.05)$. In the ART (Fig. 3B), RI mice removed adhesive spots on their right forepaws more rapidly than the control mice $(7.6 \pm 0.9$ vs. $13.07 \pm 2.1 \mathrm{sec}$; $\mathrm{P}<0.05)$. In addition, RI mice removed adhesive spots on their right forepaws quicker than those on their left $(7.6 \pm 0.9$ vs. $12.2 \pm 2.1 \mathrm{sec}$ ), although the difference was not statistically significant ( $\mathrm{P}=0.052$; Fig. 3B).

A notable reduction of ROCKII-positive staining was observed in the $\mathrm{SN}$ region of RI mice (Fig. $4 \mathrm{~A}$ and $\mathrm{B} ; \mathrm{P}<0.01$ ). TH-positive cell numbers in the SN region were markedly increased in RI mice when compared with the control mice (Fig. 4A; P<0.01). The protein expression levels of ROCKII and p-MYPT1 in the right hemisphere of RI mice was reduced by 21 and $37 \%$ compared with controls, respectively, as measured
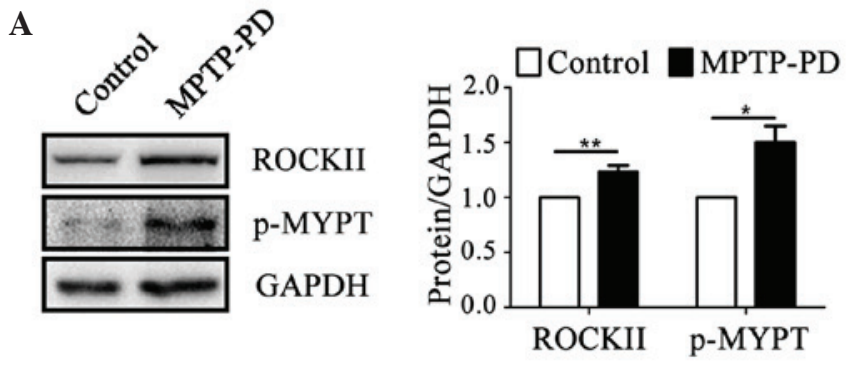

B Control

MPTP-PD
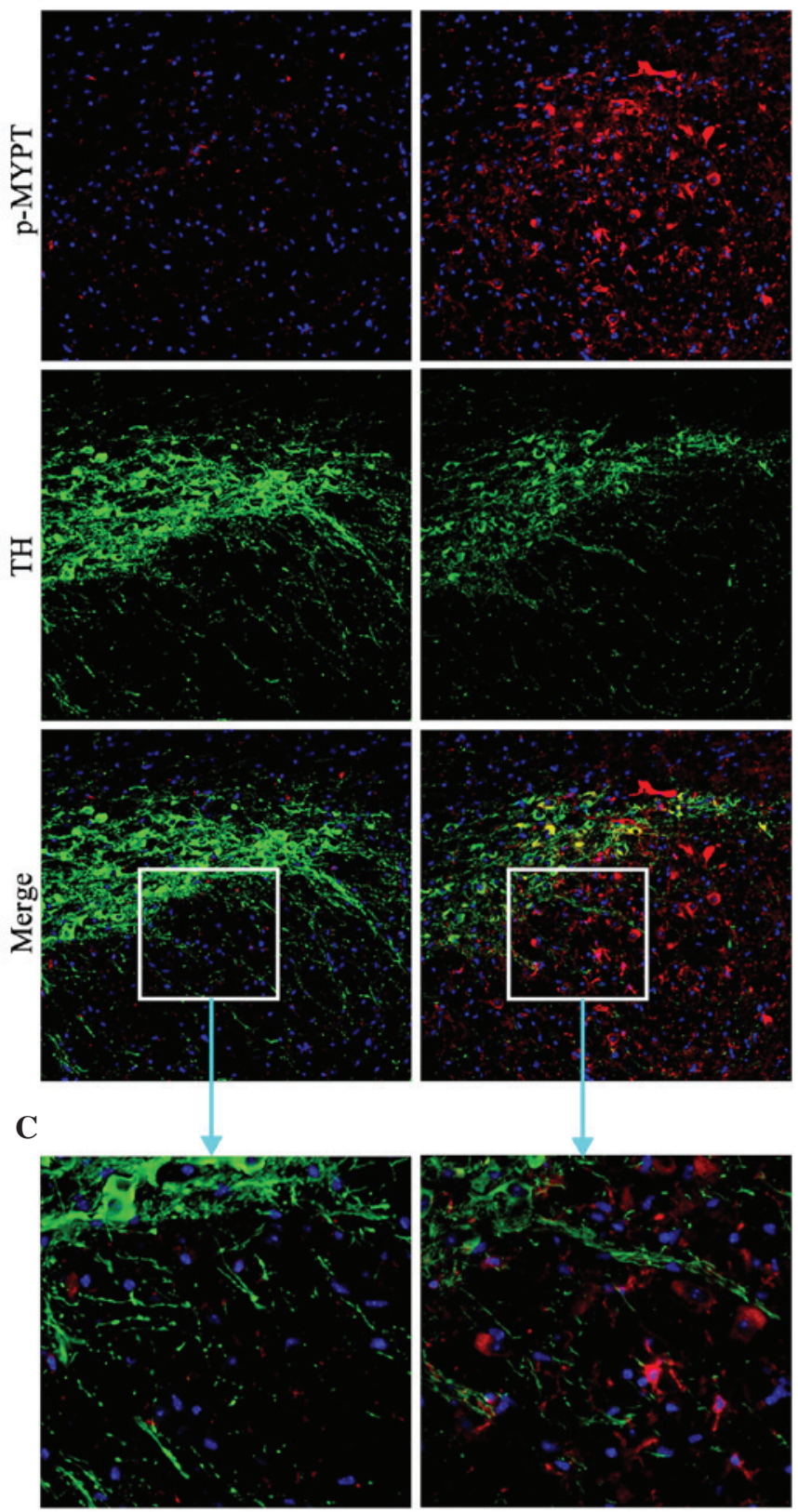

Figure 1. ROCKII protein expression level and enzyme activity were increased in the brains of mice with MPTP-induced parkinsonism. (A) The protein expression levels of ROCKII and p-MYPT increased in the brains of the MPTP-induced PD mouse model as determined by western blotting ( $\mathrm{n}=4$; ROCKII, $\mathrm{P}=0.015$; p-MYPT, $\mathrm{P}=0.001)$. (B) p-MYPT staining of the SN of MPTP-treated and control mice (magnification, x20). p-MYPT-positive cells were abundant in MPTP-treated mice compared with the control mice. A proportion of p-MYPT-positive cells expressed TH. (C) Magnification of the boxed areas in (B) (magnification, x40). ROCKII, Rho/Rho-associated, coiled-coil-containing protein kinase II; MPTP, 1-methyl-4-phenyl-1,2,3,6-tetrahydropyridine; PD, Parkinson's disease; p-MYPT, phosphorylated myosin phosphate target subunit; SN, substantia nigra; TH, tyrosine hydroxylase. 
A

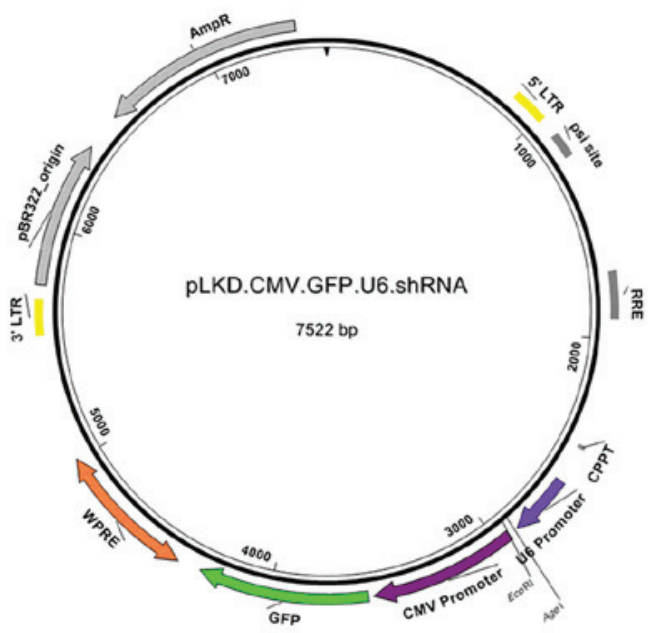

B
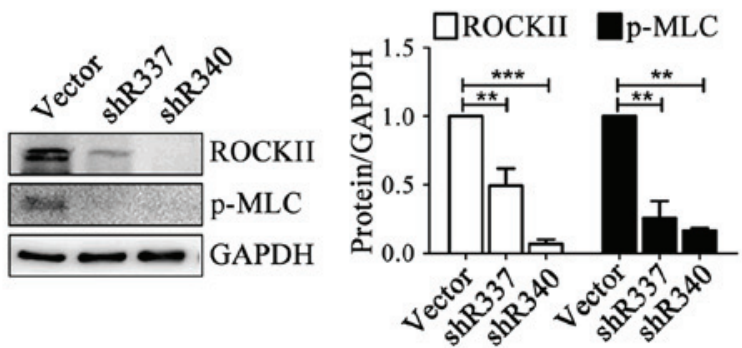
$\underset{\text { GGo CCT ATT TCC CAT GAT TCC TTC ATA TIT GC...CAC CGG TCC GCA GGT ATG }}{\stackrel{2685}{\text { Agel }}} \stackrel{2685}{2905}$

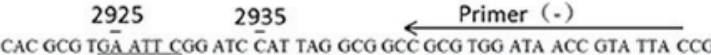
EcoRI

C
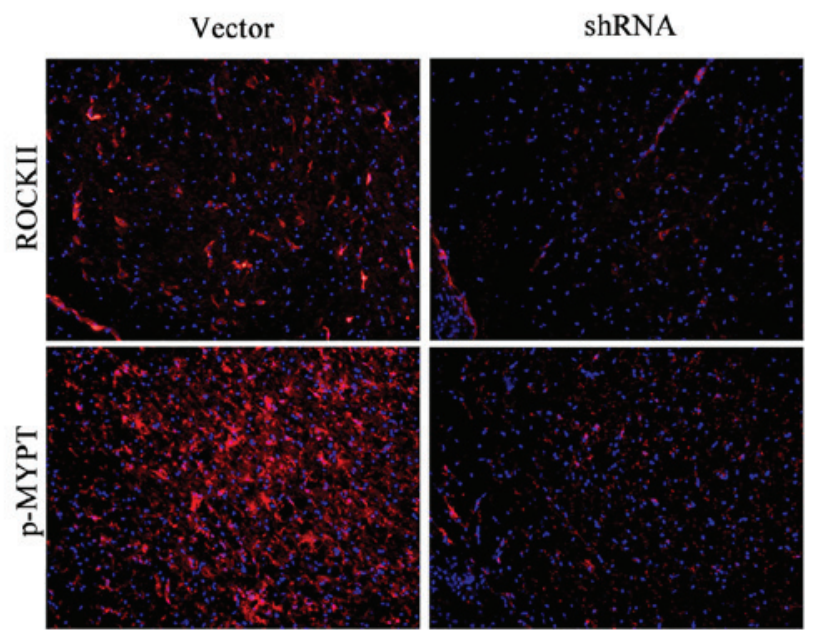

Figure 2. Construction of a lentivirus-based shRNA system and interference efficiency of anti-ROCKII shRNA in vitro and in vivo. (A) Construction schematic of the lentivirus vehicle, driven by the U6 promoter. The GFP reporter gene is driven by the CMV promoter. (B) Interference efficiencies of anti-ROCKII shRNAs in mouse BV2 microglia cells as determined by western blotting. ROCKII and p-MLC protein levels were decreased significantly following transfection with shR337 (ROCKII, $\mathrm{P}=0.007$; $\mathrm{p}-\mathrm{MLC}, \mathrm{P}=0.006$ ) and shR340 (ROCKII, $\mathrm{P}<0.001$; $\mathrm{p}-\mathrm{MLC}, \mathrm{P}=0.002$ ), compared with the empty vector ( $\mathrm{n}=3$ ). ${ }^{* *} \mathrm{P}<0.01$ and ${ }^{* * *} \mathrm{P}<0.001$. (C) Interference efficiencies of anti-ROCKII shRNAs in the brains of mice determined by fluorescence (magnification, $\left.x 20\right)$. Stereotaxic injection of the combination of shR337 and shR340 at a ratio of 1:1 into the SN area markedly reduced ROCKII and p-MYPT staining compared with the empty vector. shRNA, small hairpin RNA; ROCKII, Rho/Rho-associated, coiled-coil-containing protein kinase II; GFP, green fluorescent protein; CMV, cytomegalovirus; p-MLC, phosphorylated myosin light chain; SN, substantia nigra; p-MYPT, phosphorylated myosin phosphate target subunit.

A

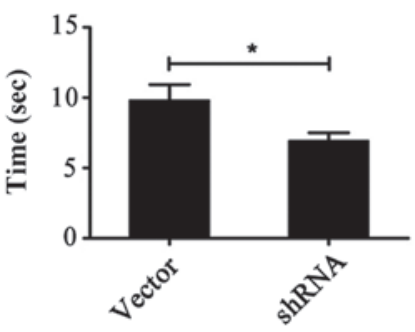

B

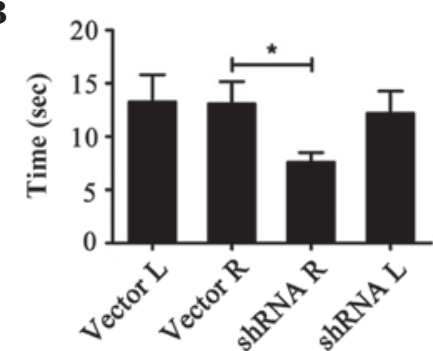

Figure 3. RI attenuated movement disturbance induced in mice by MPTP. (A) Pole test. RI mice move more rapidly than control mice ( $\mathrm{n}=8$; $\mathrm{P}=0.036$ ), (B) Adhesive removal test. RI mice removed adhesive spots on their right forepaws more quickly than the control mice $(\mathrm{n}=8 ; \mathrm{P}=0.028)$. In addition, RI mice removed adhesive spots on their right forepaws more rapidly than those on their left $(\mathrm{P}=0.052)$. The time taken for left forepaw adhesive spot removal did not differ between groups. "P<0.05. RI, Rho/Rho-associated, coiled-coil-containing protein kinase II interference; MPTP, 1-methyl-4-phenyl-1,2,3,6-tetrahydropyridine; L, left forepaw; R, right forepaw; shRNA, small hairpin RNA. 
A
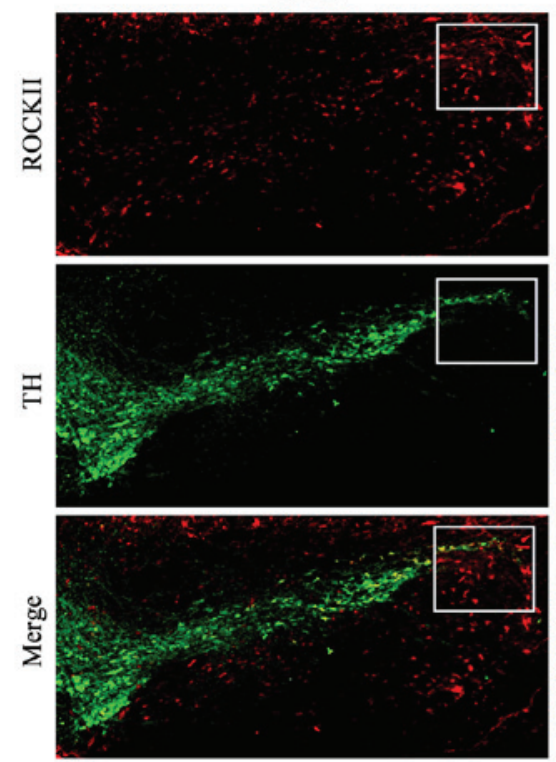

B
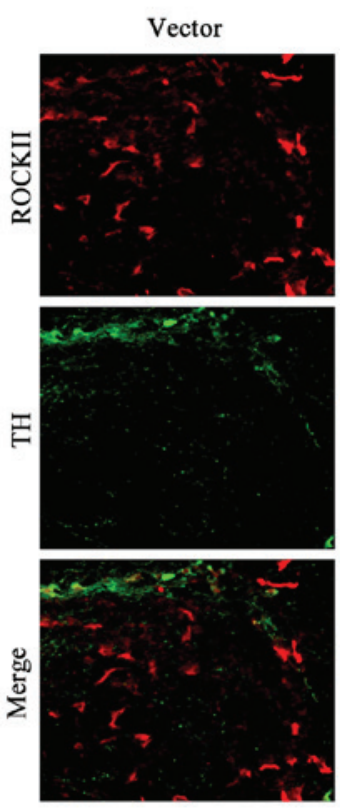
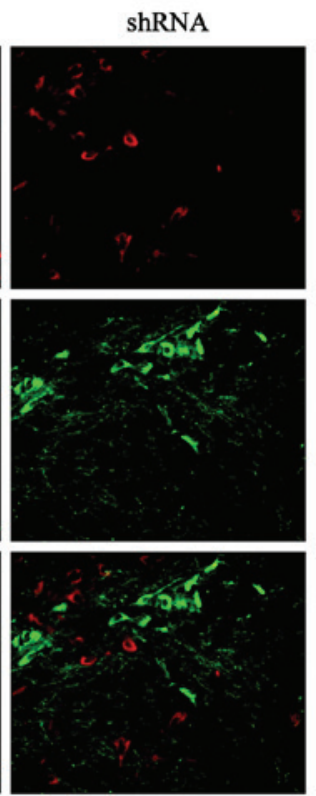

shRNA

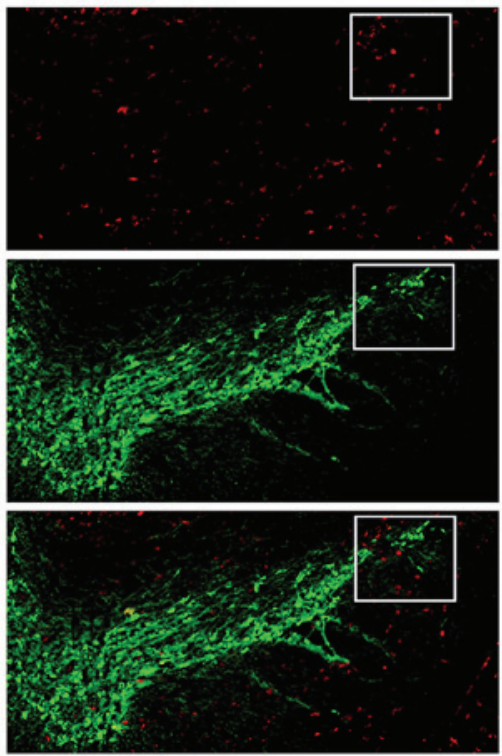

C
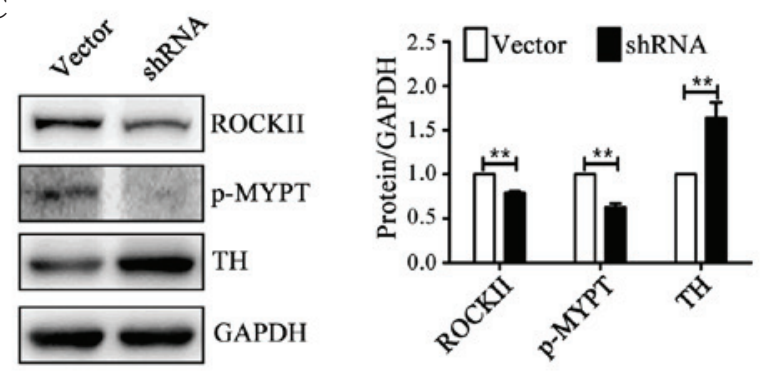

D

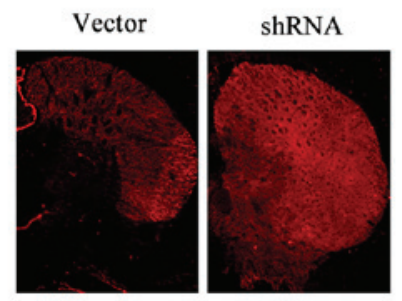

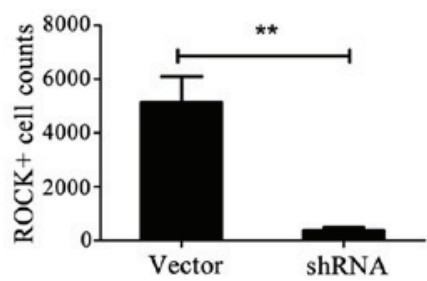

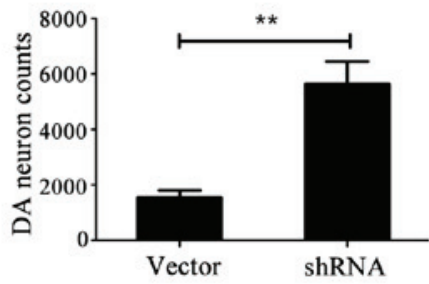

Figure 4. RI significantly inhibited MPTP-induced TH-positive neuron loss. (A) Local injection of shRNA efficiently reduced the expression level of ROCKII $(\mathrm{P}=0.007)$ and the TH-positive area at substantia nigra $(\mathrm{n}=5 ; \mathrm{P}=0.005)$ compared with the empty vector (magnification, $\mathrm{x} 20)$. (B) Magnification $(\mathrm{x} 40)$ of the boxed areas in (A). (C) The protein levels of ROCKII ( $\mathrm{P}=0.004)$ and $\mathrm{p}-\mathrm{MYPT}(\mathrm{P}=0.001)$ were significantly decreased and the protein level of TH $(\mathrm{P}=0.001)$ significantly increased in the RI group compared with empty vector controls $(\mathrm{n}=4)$. (D) TH-positive area in the striatum was increased in the RI group compared with the empty vector controls (magnification, $\mathrm{x} 20 ; \mathrm{n}=5 ; \mathrm{P}=0.005) .{ }^{* *} \mathrm{P}<0.01$. ROCKII, Rho/Rho-associated, coiled-coil-containing protein kinase II; RI, ROCKII interference; MPTP, 1-methyl-4-phenyl-1,2,3,6-tetrahydropyridine; TH, tyrosine hydroxylase; shRNA, small hairpin RNA; p-MYPT, phosphorylated myosin phosphate target subunit; DA, dopaminergic.

by western blotting $(\mathrm{P}<0.01$; Fig. $4 \mathrm{C})$. These results indicated successful interference of ROCKII. The expression level of $\mathrm{TH}$ protein in the right hemispheres of RI mice was $\sim 1.6$ times that of the control mice $(\mathrm{P}<0.01$; Fig. $4 \mathrm{C})$. In addition, the TH-positive area in the striatum was augmented in RI mice $(\mathrm{P}<0.01$; Fig. 4D). These results revealed that inhibition of ROCKII effectively improved the behavioral performance and inhibited DA neuron loss in the MPTP-induced PD mouse model. in mice with MPTP-induced parkinsonism. The probable mechanisms underlying the DA neuron protection of RI against MPTP were investigated. In the SN area of MPTP-induced parkinsonism mice, the majority of ROCKII-positive cells were co-localized with CD11b-positive microglia (Fig. 5A). As ROCKII was inhibited, the activation of CD11b-positive microglia was markedly inhibited in the $\mathrm{SN}$ area of RI mice (Fig. 5B).

It is widely accepted that activated microglia exert dual functions, and are defined as pro-inflammatory (M1) and anti-inflammatory (M2) microglia (18). Microglia in the SN region of MPTP-induced PD mice polarized toward the M1 
A
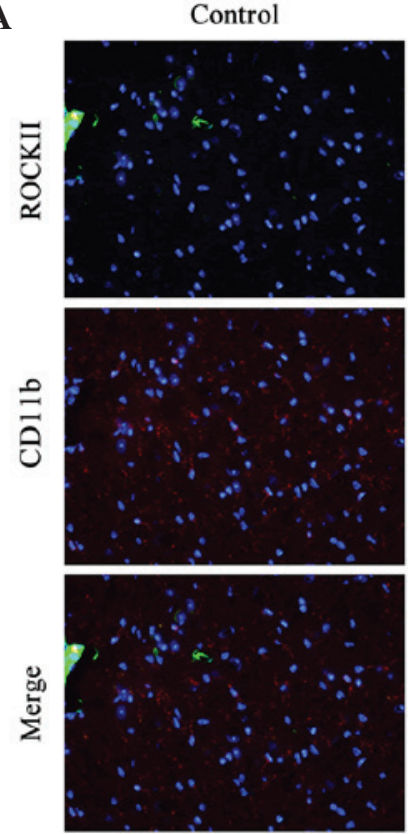

MPTP-PD
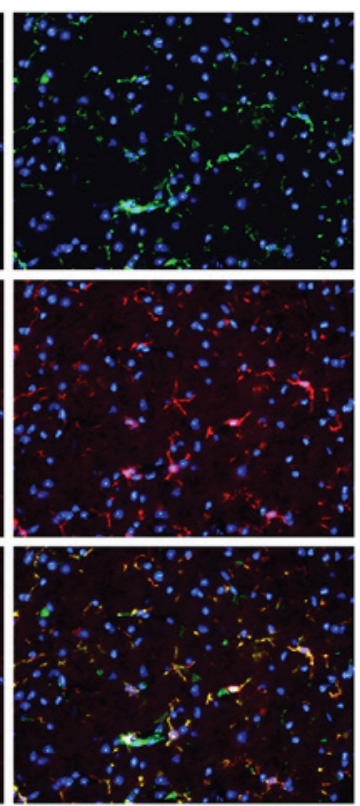

B
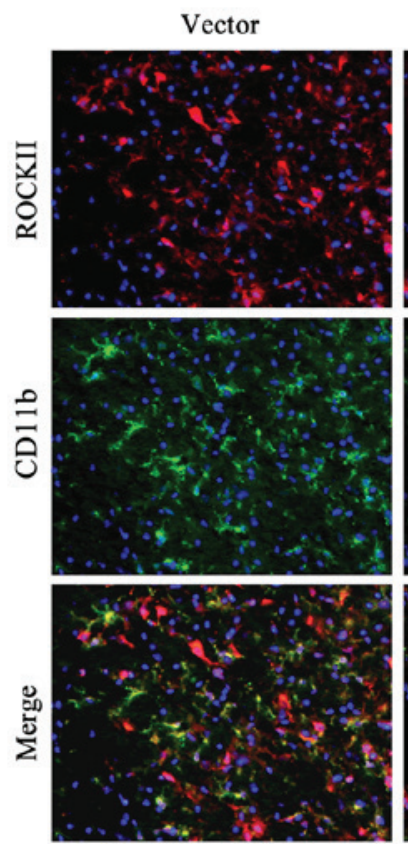

ShRNA
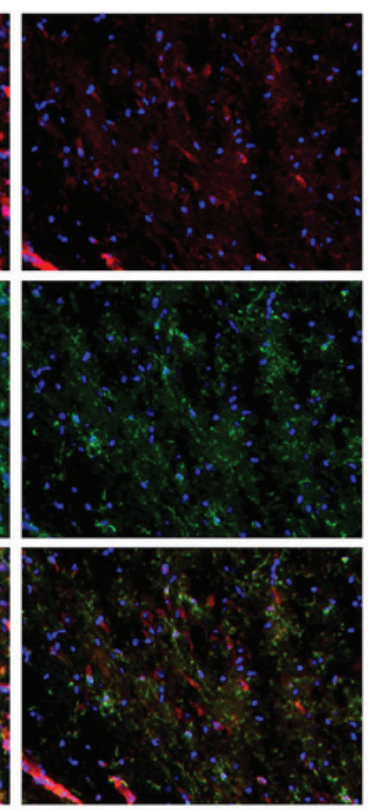

Figure 5. RI inhibited microglia activation in mice with MPTP-induced parkinsonism. (A) ROCKII-positive and CD11b-positive staining is increased in the SN in mice with MPTP-induced PD compared with the control mice (magnification x20). The majority of ROCKII-positive cells were CD11b-positive. (B) CD11b-p ositive staining declined markedly in the RI mice as ROCKII-positive staining declined (magnification, x20). ROCKII, Rho/Rho-associated, coiled-coilcontaining protein kinase II; RI, ROCKII interference; MPTP, 1-methyl-4-phenyl-1,2,3,6-tetrahydropyridine; PD, Parkinson's disease; CD11b, cluster of differentiation $11 \mathrm{~b}$; SN, substantia nigra.

subtype, expressing high levels of iNOS, TLR2 and NF- $\mathrm{B}$ (Fig. 6A). RI attenuated iNOS and TLR2 expression levels, and inhibited $\mathrm{I} \kappa \mathrm{B} / \mathrm{IKK} / \mathrm{TBK} 1 / \mathrm{NF}-\kappa \mathrm{B}$ signaling pathway activation $(\mathrm{P}<0.05$; Fig. $6 \mathrm{~B}$ and $\mathrm{C})$.

The production of IL-1 $\beta$ and IL- 6 decreased significantly $(\mathrm{P}<0.05$ and $\mathrm{P}<0.01$, respectively) in the brain of $\mathrm{RI}$ mice (Fig. 6D). However, the production of TNF- $\alpha$ did not differ significantly (Fig. 6D). These results demonstrated that the inhibition of ROCKII may contribute to the neuro-protection of DA neurons against MPTP by inhibiting the activation of $\mathrm{M} 1$ microglia in the $\mathrm{SN}$ region and the release of inflammatory cytokines.

ROCK/NF- $\kappa B$ axis-dependent anti-inflammation in BV2 microglia. Whether RI influenced the activity of the $\mathrm{NF}-\kappa \mathrm{B}$ signaling pathway and inflammatory responses was investigated in mouse BV2 microglia cell lines. As presented in Fig. 7, RI resulted in the downregulation of p-MYPT expression $(\mathrm{P}<0.01$; Fig. $7 \mathrm{~A}$ and $\mathrm{B})$, which was accompanied by a decrease in $\mathrm{NF}-\kappa \mathrm{B}$ activity $(\mathrm{P}<0.01$; Fig. $7 \mathrm{~A}$ and $\mathrm{B})$. In addition, the inhibition of iNOS indicated that inflammatory M1 microglia were suppressed $(\mathrm{P}<0.01$; Fig. $7 \mathrm{~A}$ and $\mathrm{B})$. In a previous study, microglia polarization from M1 toward M2 was associated with reduced NF- $\kappa$ B activity (18). These results, combined with the in vivo data, suggest the existence of a ROCK/NF- $\kappa \mathrm{B}$ axis.

\section{Discussion}

The expression and activity of ROCKs are elevated in neuro-degenerative diseases, such as PD (11) and AD (20). ROCK inhibitors, including Y27632 or fasudil, have been shown to protect neurons from degeneration in animal models of these diseases, although the underlying mechanism remains unclear $(10,20)$. It has been reported that ROCK activation increases angiotensin type-1 receptor expression in neurons, which promotes the neuro-degenerative process (21).

Previous studies have demonstrated that ROCKs are involved in inflammation. ROCK activation increased the permeability of endothelial cells and promoted lymphocyte infiltration (22). In addition, ROCK activation enhanced the phagocytic activity of macrophages (23), and the release of reactive oxygen species and pro-inflammatory cytokines by inflammatory cells (24). The neuro-protective effects of fasudil, a ROCK inhibitor $(4,25)$ may be partly mediated through inhibiting neuro-inflammation. In a previous study, fasudil exhibited therapeutic potential in experimental autoimmune encephalomyelitis (26). Fasudil rebalanced T helper 17 and regulatory $\mathrm{T}$ cells, induced microglial polarization towards M2, and inhibited inflammatory responses (26).

Neuro-inflammation has been observed in autopsies of PD patients (27). Lymphocyte infiltration, inappropriate microglia activation $(27,28)$, upregulation of pro-inflammatory cytokines, including IL-1 $\beta$, IL-6 and TNF- $\alpha$ (29-31), and upregulation of inflammation-associated signaling molecules, including iNOS and NF-кB (31-33) have all been observed in the $\mathrm{SN}$ of the brain. In addition, imaging has provided evidence of microglial activation in early PD patients $(34,35)$. Furthermore, corresponding inflammatory conditions have been described in toxin-based parkinsonism models, with high levels of microglia in the SN (36-40). DA neurons are particularly vulnerable to the inflammatory microenvironment, and these responses may be crucial in aggravating DA degeneration in the $\mathrm{SN}$ region (41). Thus, it is proposed that 
A
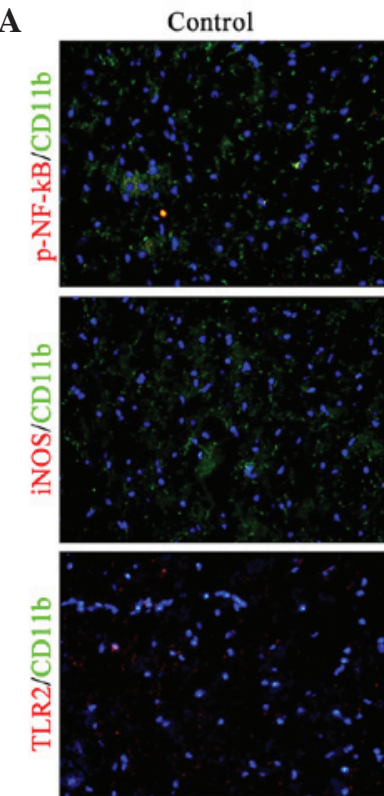

C

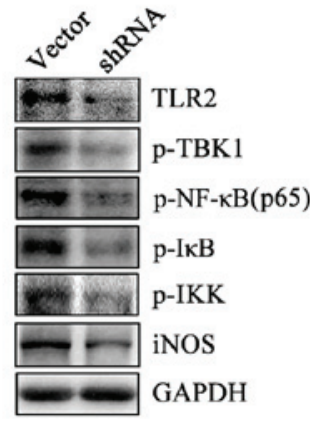

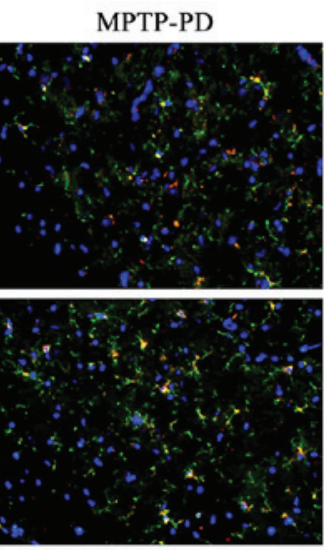
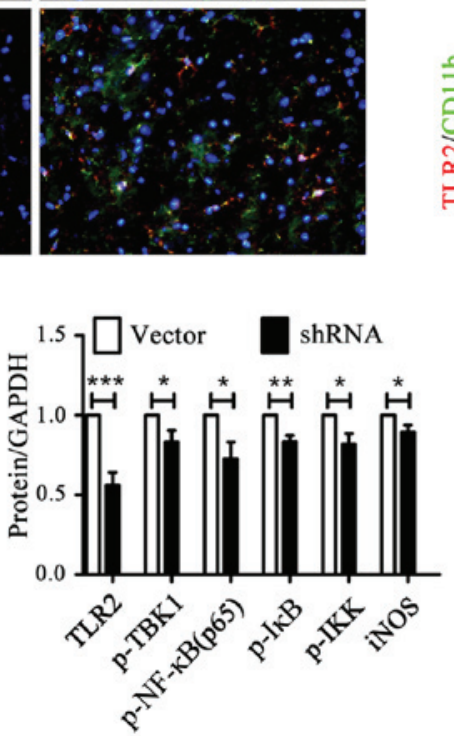

B
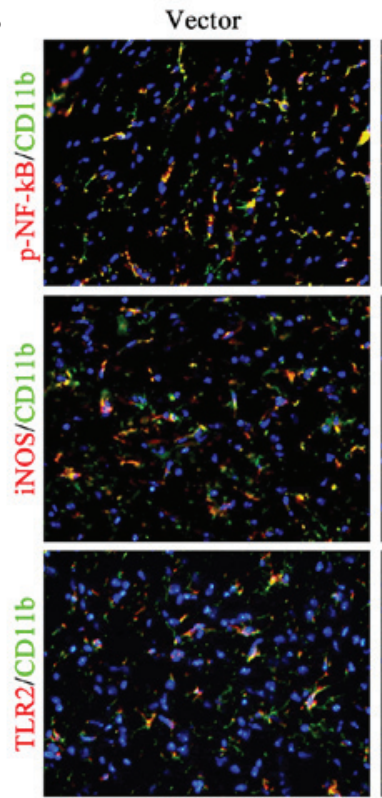

D
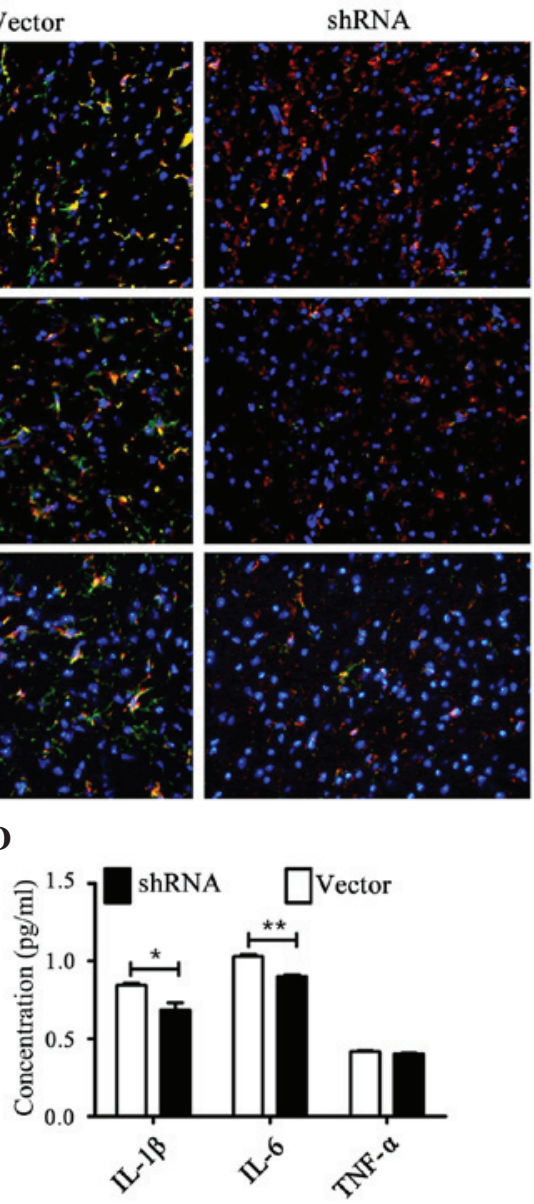

Figure 6. RI inhibited microglia activation in mice with MPTP-induced parkinsonism by inhibiting NF-kB activation. (A) p-p65, the activated form of NF-kB, increased significantly in the SN of MPTP-treated mice and was present in CD11b-positive cells (magnification, x20). In addition, iNOS and TLR2 were upregulated in MPTP-treated mice and expressed by activated microglia. (B) p-p65, iNOS and TLR2 all decreased in the RI group as microglia activation was inhibited (magnification, $\mathrm{x} 20)$. (C) p-p65 ( $\mathrm{P}=0.015)$, iNOS $(\mathrm{P}=0.049)$ and TLR2 ( $\mathrm{P}<0.001)$ were downregulated in RI mice. In addition, $\mathrm{p}-\mathrm{IKK}(\mathrm{P}=0.028)$, $\mathrm{p}-\mathrm{I \kappa B}(\mathrm{P}=0.002)$ and $\mathrm{p}-\mathrm{TBK} 1(\mathrm{P}=0.035)$ expression levels were inhibited in RI mice $(\mathrm{n}=4)$. ( $\mathrm{D})$ The expression levels of IL-1 $(\mathrm{P}=0.028)$ and IL-6 $(\mathrm{P}=0.002)$ were elevated significantly in RI mice compared with the control mice. No difference in TNF- $\alpha$ expression was detected. ${ }^{*} \mathrm{P}<0.05,{ }^{* *} \mathrm{P}<0.01,{ }^{* * *} \mathrm{P}<0.001$. RI, Rho/Rho-associated, coiled-coil-containing protein kinase II interference; MPTP, 1-methyl-4-phenyl-1,2,3,6-tetrahydropyridine; PD, Parkinson's disease;

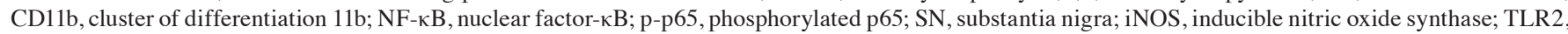
Toll-like receptor 2; p-IKK, phosphorylated inhibitor of $\kappa \mathrm{B}$ kinase; $\mathrm{p}-\mathrm{I} \mathrm{B}$, phosphorylated inhibitor of $\kappa \mathrm{B}$; $\mathrm{p}$-TBK1, phosphorylated TANK-binding kinase 1; IL, interleukin; TNF- $\alpha$, tumor necrosis factor $\alpha$; shRNA, small hairpin RNA.

$\mathbf{A}$

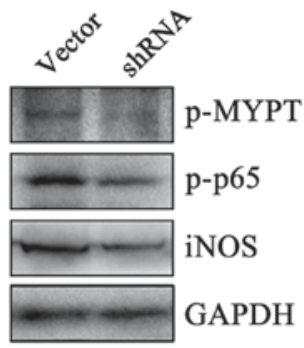

B

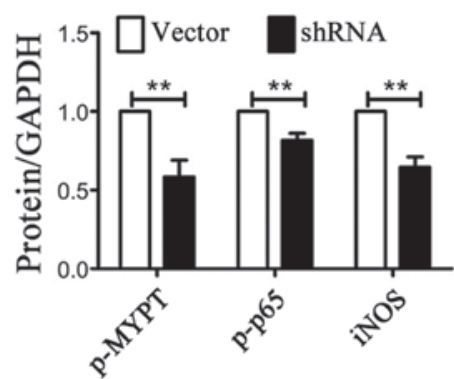

Figure 7. RI inhibited microglia activation in mouse BV2 microglia cells by inhibiting the NF-kB signaling pathway. (A) Representative bands of a western blot assay to detect p-MYPT, p-p65 and iNOS. (B) Expression levels of p-MYPT ( $\mathrm{P}=0.008)$, p-p65 ( $\mathrm{P}=0.005)$ and iNOS ( $\mathrm{P}=0.007)$ were significantly decreased in RI mice compared with the control mice. ${ }^{* *} \mathrm{P}<0.01$. RI, Rho/Rho-associated, coiled-coil-containing protein kinase II interference; NF- $\mathrm{kB}$, nuclear factor- $\mathrm{kB}$; p-MYPT, phosphorylated myosin phosphate target subunit; p-p65, phosphorylated p65; iNOS, inducible nitric oxide synthase; shRNA, small hairpin RNA.

activation of microglia is a risk factor triggering the onset of a cascade of events leading to progressive degeneration of DA neurons.
In our previous study, fasudil skewed LPS-stimulated M1 microglia towards the M2 subtype, expressing reduced NF- $\mathrm{KB}$ activity and inflammatory cytokine levels, including IL-1 $\beta$, 
IL-6 and TNF- $\alpha$ (18). In the present study, RI also inhibited M1 microglia activation, and the expression of iNOS and additional pro-inflammatory molecules in the SN region of MPTP-treated mice, as well as in mouse BV2 microglia cells. This internal environmental change may have a neuro-protective effect on DA neurons. In addition, a recent study demonstrated that medium from microglia stimulated with LPS and fasudil exerted a neuro-protective effect on rat PC12 neurons, suggesting that inhibition of ROCK contributes to the survival of DA neurons (18). As microglial activation is aggressively initiated in early PD (34) and remains stable for years (35), it may be essential to consider ROCK inhibition in the early stages of PD to prevent glial-mediated inflammation and neuronal elimination. Given that MPTP/MPP+ directly damages DA neurons in vivo, whether the shRNA directly inhibited ROCKII activity in DA neurons and thus protected them from apoptosis, or whether this effect was an indirect result of the inhibited pro-inflammatory effect of microglia, could not be established in the current study. However, experiments are planned to separately analyze neuron-targeted and microglia-targeted shRNA systems to further investigate the underlying mechanisms.

The greatest problem with ROCK inhibitors is that neither Y27632 nor fasudil exclusively inhibit ROCKs. In addition, they slightly influence PKN, MSK1, MAPK1b and PKA, and moderately regulate AMPK and PHK (12). It has thus been difficult to accurately evaluate the contribution of ROCKs to the process of neuro-degeneration and investigate the neuro-protective effect of ROCK inhibitors in neuro-degenerative diseases. The present study used RNA interference to specifically inhibit ROCKII expression and verify its role in an MPTP-induced PD mouse model.

In conclusion, the results of the present study indicate that ROCKII may serve a crucial role in MPTP-induced parkinsonism in mice. In addition, the results demonstrate that RI is a promising therapeutic target that inhibits the activation of inflammatory microglia in the $\mathrm{SN}$ region.

\section{Acknowledgements}

The present study was supported by grants from the National Foundation of Natural Science of China (grant nos. 81070956 and 81371414).

\section{References}

1. Dawson TM and Dawson VL: Molecular pathways of neurodegeneration in Parkinson's disease. Science 302: 819-822, 2003.

2. Fox R, Nhan TQ, Law GL, Morris DR, Liles WC and Schwartz SM: PSGL-1 and mTOR regulate translation of ROCK-1 and physiological functions of macrophages. EMBO J 26: 505-515, 2007.

3. Yoneda A, Multhaupt HA and Couchman JR: The Rho kinases I and II regulate different aspects of myosin II activity. J Cell Biol 170: 443-453, 2005.

4. Watanabe K, Ueno M, Kamiya D, Nishiyama A, Matsumura M, Wataya T, Takahashi JB, Nishikawa S, Nishikawa S, Muguruma K and Sasai Y: A ROCK inhibitor permits survival of dissociated human embryonic stem cells. Nat Biotechnol 25: 681-686, 2007.

5. Mong PY and Wang Q: Activation of Rho kinase isoforms in lung endothelial cells during inflammation. J Immunol 182: 2385-2394, 2009.

6. Aihara M, Dobashi K, Iizuka K, Nakazawa T and Mori M: Comparison of effects of Y-27632 and Isoproterenol on release of cytokines from human peripheral $\mathrm{T}$ cells. Int Immunopharmacol 3: 1619-1625, 2003.
7. Aihara M, Dobashi K, Iizuka K, Nakazawa T and Mori M: Effect of Y-27632 on release of cytokines from peripheral T cells in asthmatic patients and normal subjects. Int Immunopharmacol 4: 557-561, 2004

8. Henry PJ, Mann TS and Goldie RG: A rho kinase inhibitor, Y-27632 inhibits pulmonary eosinophilia, bronchoconstriction and airways hyperresponsiveness in allergic mice. Pulm Pharmacol Ther 18: 67-74, 2005.

9. Song Y, Chen X, Wang LY, Gao W and Zhu MJ: Rho kinase inhibitor fasudil protects against $\beta$-amyloid-induced hippocampal neurodegeneration in rats. CNS Neurosci Ther 19: 603-610, 2013.

10. Villar-Cheda B, Dominguez-Meijide A, Joglar B, Rodriguez-Perez AI, Guerra MJ and Labandeira-Garcia JL: Involvement of microglial RhoA/Rho-kinase pathway activation in the dopaminergic neuron death. Role of angiotensin via angiotensin type 1 receptors. Neurobiol Dis 47: 268-279, 2012.

11. Tönges L, Frank T, Tatenhorst L, Saal KA, Koch JC, Szego ÉM, Bähr M, Weishaupt JH and Lingor P: Inhibition of rho kinase enhances survival of dopaminergic neurons and attenuates axonal loss in a mouse model of Parkinson's disease. Brain 135: 3355-3370, 2012.

12. Mueller BK, Mack H and Teusch N: Rho kinase, a promising drug target for neurological disorders. Nat Rev Drug Discov 4: 387-398, 2005.

13. Tokuoka H, Muramatsu S, Sumi-Ichinose C, Sakane H, Kojima M, Aso Y, Nomura T, Metzger D and Ichinose H: Compensatory regulation of dopamine after ablation of the tyrosine hydroxylase gene in the nigrostriatal projection. J Biol Chem 286: 43549-43558, 2011.

14. Matsuura K, Kabuto H, Makino H and Ogawa N: Pole test is a useful method for evaluating the mouse movement disorder caused by striatal dopamine depletion. J Neurosci Methods 73: 45-48, 1997.

15. Bouet V, Boulouard M, Toutain J, Divoux D, Bernaudin M, Schumann-Bard P and Freret T: The adhesive removal test: A sensitive method to assess sensorimotor deficits in mice. Nat Protoc 4: 1560-1564, 2009.

16. Ojo B, Rezaie P, Gabbott PL, Cowely TR, Medvedev NI, Lynch MA and Stewart MG: A neural cell adhesion molecule-derived peptide, FGL, attenuates glial cell activation in the aged hippocampus. Exp Neurol 232: 318-328, 2011.

17. Zaborszky L and Vadasz C: The midbrain dopaminergic system: Anatomy and genetic variation in dopamine neuron number of inbred mouse strains. Behav Genet 31: 47-59, 2001.

18. Zhang H, Li Y, Yu J, Guo M, Meng J, Liu C, Xie Y, Feng L, Xiao B and Ma C: Rho kinase inhibitor fasudil regulates microglia polarization and function. Neuroimmunomodulation 20: 313-322, 2013.

19. Grassie ME, Moffat LD, Walsh MP and MacDonald JA: The myosin phosphatase targeting protein (MYPT) family: A regulated mechanism for achieving substrate specificity of the catalytic subunit of protein phosphatase type 1ס. Arch Biochem Biophys 510: 147-159, 2011.

20. Herskowitz JH, Feng Y, Mattheyses AL, Hales CM, Higginbotham LA, Duong DM, Montine TJ, Troncoso JC, Thambisetty M, Seyfried NT, et al: Pharmacologic inhibition of ROCK2 suppresses amyloid- $\beta$ production in an Alzheimer's disease mouse model. J Neurosci 33: 19086-19098, 2013.

21. Rodriguez-Perez AI, Dominguez-Meijide A, Lanciego JL, Guerra MJ and Labandeira-Garcia JL: Inhibition of Rho kinase mediates the neuroprotective effects of estrogen in the MPTP model of Parkinson's disease. Neurobiol Dis 58: 209-219, 2013.

22. Noma K, Kihara Y and Higashi Y: Striking crosstalk of ROCK signaling with endothelial function. J Cardiol 60: 1-6, 2012.

23. Barcia C, Ros CM, Annese V, Carrillo-de Sauvage MA, Ros-Bernal F, Gómez A, Yuste JE, Campuzano CM, de Pablos V, Fernandez-Villalba E and Herrero MT: ROCK/Cdc42-mediated microglial motility and gliapse formation lead to phagocytosis of degenerating dopaminergic neurons in vivo. Sci Rep 2: 809, 2012.

24. Satoh K, Fukumoto Y and Shimokawa H: Rho-kinase: Important new therapeutic target in cardiovascular diseases. Am J Physiol Heart Circ Physiol 301: H287-H296, 2011.

25. Wu KY, Hengst U, Cox LJ, Macosko EZ, Jeromin A, Urquhart ER and Jaffrey SR: Local translation of RhoA regulates growth cone collapse. Nature 436: 1020-1024, 2005.

26. Liu CY, Guo SD, Yu JZ, Li YH, Zhang H, Feng L, Chai Z, Yuan HJ, Yang WF, Feng QJ, et al: Fasudil mediates cell therapy of EAE by immunomodulating encephalomyelitic $\mathrm{T}$ cells and macrophages. Eur J Immunol 45: 142-152, 2015. 
27. McGeer PL,ItagakiS, Boyes BEand McGeer EG: Reactive microglia are positive for HLA-DR in the substantia nigra of Parkinson's and Alzheimer's disease brains. Neurology 38: 1285-1291, 1988.

28. Brochard V, Combadière B, Prigent A, Laouar Y, Perrin A, Beray-Berthat V, Bonduelle O, Alvarez-Fischer D, Callebert J, Launay JM, et al: Infiltration of CD4+ lymphocytes into the brain contributes to neurodegeneration in a mouse model of Parkinson disease. J Clin Invest 119: 182-192, 2009.

29. Boka G, Anglade P, Wallach D, Javoy-Agid F, Agid Y and Hirsch EC: Immunocytochemical analysis of tumor necrosis factor and its receptors in Parkinson's disease. Neurosci Lett 172: 151-154, 1994.

30. Mogi M, Harada M, Riederer P, Narabayashi H, Fujita K and Nagatsu T: Tumor necrosis factor-alpha (TNF-alpha) increases both in the brain and in the cerebrospinal fluid from parkinsonian patients. Neurosci Lett 165: 208-210, 1994.

31. Mogi M, Harada M, Kondo T, Riederer P, Inagaki H, Minami M and Nagatsu T: Interleukin-1 beta, interleukin-6, epidermal growth factor and transforming growth factor-alpha are elevated in the brain from parkinsonian patients. Neurosci Lett 180 : 147-150, 1994.

32. Mogi M, Kondo T, Mizuno $\mathrm{Y}$ and Nagatsu T: p53 protein, interferon-gamma, and NF-kappaB levels are elevated in the parkinsonian brain. Neurosci Lett 414: 94-97, 2007.

33. Knott C, Stern G and Wilkin GP: Inflammatory regulators in Parkinson's disease: iNOS, lipocortin-1, and cyclooxygenases-1 and -2. Mol Cell Neurosci 16: 724-739, 2000.

34. Ouchi Y, Yoshikawa E, Sekine Y, Futatsubashi M, Kanno T, Ogusu T and Torizuka T: Microglial activation and dopamine terminal loss in early Parkinson's disease. Ann Neurol 57: 168-175, 2005.
35. Gerhard A, Pavese N, Hotton G, Turkheimer F, Es M, Hammers A, Eggert K, Oertel W, Banati RB and Brooks DJ: In vivo imaging of microglial activation with $[11 C](\mathrm{R})-\mathrm{PK} 11195$ PET in idiopathic Parkinson's disease. Neurobiol Dis 21: 404-412, 2006.

36. Liberatore GT, Jackson-Lewis V, Vukosavic S, Mandir AS, Vila M, McAuliffe WG, Dawson VL, Dawson TM and Przedborski S: Inducible nitric oxide synthase stimulates dopaminergic neurodegeneration in the MPTP model of Parkinson disease. Nat Med 5: 1403-1409, 1999.

37. Teismann P, Tieu K, Choi DK, Wu DC, Naini A, Hunot S, Vila M, Jackson-Lewis V and Przedborski S: Cyclooxygenase-2 is instrumental in Parkinson's disease neurodegeneration. Proc Natl Acad Sci USA 100: 5473-5478, 2003.

38. Cicchetti F, Lapointe N, Roberge-Tremblay A, Saint-Pierre M, Jimenez L, Ficke BW and Gross RE: Systemic exposure to paraquat and maneb models early Parkinson's disease in young adult rats. Neurobiol Dis 20: 360-371, 2005.

39. Noelker C, Morel L, Lescot T, Osterloh A, Alvarez-Fischer D, Breloer M, Henze C, Depboylu C, Skrzydelski D, Michel PP, et al: Toll like receptor 4 mediates cell death in a mouse MPTP model of Parkinson disease. Sci Rep 3: 1393, 2013.

40. Kim WG, Mohney RP, Wilson B, Jeohn GH, Liu B and Hong JS: Regional difference in susceptibility to lipopolysaccharide-induced neurotoxicity in the rat brain: Role of microglia. J Neurosci 20: 6309-6316, 2000.

41. Wang C, Wang H, Luo J, Hu Y, Wei L, Duan M and $\mathrm{He} H$ : Selenium deficiency impairs host innate immune response and induces susceptibility to Listeria monocy togenes infection. BMC Immunol 10: 55, 2009. 